\title{
The Risk of Nephrolithiasis Among Patients With Ankylosing Spondylitis: A Population-Based Cohort Study
}

\author{
Mu Tsun SHIH, ${ }^{1}$ Shou Hung TANG, ${ }^{1}$ Tai Lung CHA, ${ }^{1}$ Sheng Tang WU, ${ }^{1}$ \\ Jen Huai CHIANG, ${ }^{2}$ Wen Chi $\mathrm{CHEN}^{2,3}$ \\ ${ }^{1}$ Division of Urology, Department of Surgery, Tri-service General Hospital, National Defence Medical Center, Taipei City, Taiwan \\ ${ }^{2}$ Graduate Institute of Integrated Medicine, College of Chinese Medicine, College of Medicine, Research Center for Chinese Medicine \& \\ Acupuncture, China Medical University, Management Office for Health Data, China Medical University Hospital, Taichung City, Taiwan \\ ${ }^{3}$ Sex Hormone Research Center, Departments of Obstetrics and Gynecology, Urology, and Medical Research, \\ China Medical University Hospital, Taichung City, Taiwan
}

ABSTRACT

Objectives: This study aims to investigate the correlation of ankylosing spondylitis (AS) with nephrolithiasis by performing a nationwide populationbased cohort study.

Patients and methods: The data used in this retrospective cohort study were collected from the Taiwan National Health Insurance Research database. The study included a total of 3,334 AS patients (1,914 males, 1,420 females; mean age $44.51 \pm 16.83$ years; range 22 to 79 years) and 13,336 non-AS patients (7,656 males, 5,680 females; mean age $44.27 \pm 17.01$ years; range 21 to 78 years) who had been followed-up on an average of 6.78 years and 6.75 years, respectively, between January 2000 and December 2008.

Results: The percentage of newly diagnosed nephrolithiasis was $4.8 \%$ in all study subjects. However, the percentages of newly diagnosed nephrolithiasis were $5.76 \%$ and $4.58 \%$ in the AS and non-AS cohorts, respectively. After adjusting the patients' sex, age, urbanization level, and comorbidities, the hazard ratio obtained from our multivariable Cox model was 1.19.

Conclusion: Our study findings indicate that patients with AS are more likely to develop nephrolithiasis than non-AS patients.

Keywords: Ankylosing spondylitis; comorbidities; nephrolithiasis.

Ankylosing spondylitis (AS) is known as a chronic inflammatory rheumatic disorder which mainly affects the axial skeleton such as spinal and sacroiliac joints. In addition, AS sometimes affects the peripheral joints and nonarticular structures such as in associated diseases including acute uveitis, psoriasis, and inflammatory bowel disease. ${ }^{1}$ The prevalence of AS in Taiwan is approximately $0.2 \%$ to $0.3 \%$ of its overall population, ${ }^{2}$ which is similar to that of Europe as well as North America. $^{3}$ On the other hand, nephrolithiasis is known as a common urological disease with an average prevalence of $10 \%$ to $15 \%$ in world population. ${ }^{4}$ Typical symptoms include renal colic and hematuria, while other symptoms such as radiating pain, and gastrointestinal and lower urinary tract symptoms are also common. ${ }^{5}$

Most of the urinary stones (over 80\%) are known to be calcium containing. Altered calcium metabolism and hyperuricemia have often been observed among severe AS patients or those with extensive disease duration. ${ }^{6}$ Since AS patients 
tend to have a higher risk of inflammatory bowel disease, this further leads to patients' increased absorption of calcium and oxalate. ${ }^{7}$ Overall, these circumstances raise our concern whether AS should considered as one of the many risk factors which might lead to nephrolithiasis.

Over the years, various population-based studies using the Taiwan National Health Insurance Research database (NHIRD) have been conducted to validate the correlations between nephrolithiasis and diseases such as osteoporosis, diabetes mellitus, and migraine. ${ }^{8-10}$ Therefore, in this study, we aimed to investigate the correlation of AS with nephrolithiasis by performing a nationwide population-based cohort study.

\section{PATIENTS AND METHODS}

This study used reimbursement claims data from the Taiwan NHIRD. Taiwan's National Health Insurance program, which has been implemented by the government since March 1996, provides comprehensive health care to almost all Taiwanese citizens, with a coverage rate of more than $99 \%$ of Taiwan's entire population. The National Health Research Institute (NHRI) of Taiwan manages and publicly releases multiple National Health Insurance databases for research purposes. While the NHRI not only manages data from 19 medical centers, it also covers data collected from other regional hospitals, district hospitals as well as those clinics throughout Taiwan. These databases include information such as basic patient characteristics, date of visit, diagnoses codes for the International Classification of Diseases, Ninth Revision, Clinical Modification (ICD-9-CM) codes, detailed claims data for examinations, and disease management for all admitted patients and outpatients. The Longitudinal Health Insurance Database 2000 comprises a random sample of one million subjects from the NHIRD longitudinally linked data available from 1996 to 2011. Data sets of NHRI from 2000 to 2011 were selected to be used as our research database in this study. Furthermore, this study was approved by the Institutional Review Board of China Medical University in central Taiwan (CMUH104REC2-115). The study protocol was approved by the Ethics Committee of China Medical University as mentioned as Institutional Review Board of
China Medical University. A written informed consent was obtained from each patient. The study was conducted in accordance with the principles of the Declaration of Helsinki.

We conducted this population-based study at China Medical University Hospital between January 2000 and December 2011. The study included two cohorts: a total of 3,334 AS patients (1,914 males, 1,420 females; mean age $44.51 \pm 16.83$ years; range 22 to 79 years) and age-, sex- and index year-matched (ratio, 1:4) 13,336 non-AS patients (7,656 males, 5,680 females; mean age 44.27 \pm 17.01 years; range 21 to 78 years) who had been followed-up on an average of 6.78 years and 6.75 years, respectively, between January 2000 and December 2008. The population with AS was identified by code 720.0 in the ICD-9-CM. Population with AS should have had at least two claimed diagnoses. The index date was defined as the new diagnosis date for AS.

The comparison cohort, which referred to the non-AS population in this study, comprised subjects who were randomly selected from the same data used above. Each patient with newly diagnosed AS in the NHRI database was further pair-matched with three parameters which included the identical age (per 5 years), sex, and index year. The index date for non-AS was randomly assigned from January $1^{\text {st }}, 2000$ to December $31^{\text {st }}, 2008$, in accordance with the index date distribution of the AS cohort. Furthermore, the independent parameter "urbanization level" was used to categorize patients' registered residential areas within the NHRI database. As noted, there were five levels of urbanization in our study, which was calculated by incorporating variables including population density (people $/ \mathrm{km}^{2}$ ), population ratio of different education levels, population ratio of elderly, population ratio of people of agriculture workers, and the number of physicians per 100,000 people. ${ }^{11}$

In addition, for both the AS and non-AS cohorts, patients with nephrolithiasis (ICD-9-CM: 592 or 274.11) history before index date, those aged lower than 20, and those with incomplete information of age and sex were excluded.

New diagnosis of nephrolithiasis (ICD-9-CM: 592 or 274.11) in at least two records of outpatient or inpatient visits after the index date 
was defined as the primary outcome, while the time of follow-up started from the index date and ended on either the date with new diagnosis of nephrolithiasis or on December 31 2011.

Demographic factors included age and sex. Age was divided into three groups: 20 to 39 years, 40 to 64 years, and those over 65 years. Comorbidity diseases were identified from the disease records in ICD-9-CM from outpatient and inpatient visits. Diabetes mellitus (ICD-9-CM: 250; A code: A181), hypertension (ICD-9-CM: 401-405; A code: A260, A269), hyperlipidemia (ICD-9-CM: 272; A code: A182), gout (ICD-9-CM: 274), coronary heart disease (ICD-9-CM: 410-414, 429.2; A code: A279), chronic kidney disease (ICD-9-CM: 585), and osteoporosis (ICD-9-CM: 733.0) were considered as comorbid medical disorders. If these diagnostic codes were used in two or more claims before the index date, they were recorded as comorbidities.

\section{Statistical analysis}

Comparisons between the groups were performed by using the Pearson's chi-square test to examine the difference between AS and non-AS cohorts. We then evaluated the cumulative risk of nephrolithiasis for both cohorts by using the Kaplan-Meier method, and the significance of the cumulative risk curves was assessed by using the log-rank test. The Cox's proportional hazards model was used to estimate the hazards ratio for the progression of outcome. In addition, the Cox proportional hazard model was also used to calculate the 95\% confidence interval (CI) of nephrolithiasis compared between AS and non-AS cohorts. All analyses were carried out with the SAS statistical software (version 9.4 for Windows; SAS Institute, Inc., Cary, NC, USA). Statistical significance was determined as $p<0.05$.

\section{RESULTS}

Table 1 shows baseline characteristics of study participants according to the status of AS. There were more male participants than females (57.41\% vs $42.59 \%)$. The mean age of subjects with AS was $44.51 \pm 16.83$ years. The mean follow-up periods for AS and non-AS patients were 6.78 years (median, 6.87 years) and 6.75 years (median, 6.92 years), respectively. The prevalence of AS was positively associated with urbanization level: the higher the urbanization level, the higher the prevalence of AS. The prevalence of diabetes mellitus, hypertension, hyperlipidemia, gout,

Table 1. Demographic characteristics and comorbidities in patients with and without ankylosing spondylitis

\begin{tabular}{|c|c|c|c|c|c|c|c|}
\hline & \multicolumn{3}{|c|}{ Non-AS patients $(\mathrm{n}=13,336)$} & \multicolumn{3}{|c|}{ AS patients $(n=3,334)$} & \multirow[b]{2}{*}{$p \dagger$} \\
\hline & $\mathrm{n}$ & $\%$ & Mean \pm SD & $\mathrm{n}$ & $\%$ & Mean \pm SD & \\
\hline Sex & & & & & & & 0.99 \\
\hline Female & 5680 & 42.59 & & 1420 & 42.59 & & \\
\hline Male & 7656 & 57.41 & & 1914 & 57.41 & & \\
\hline Age (years) & & & $44.27 \pm 17.01$ & & & $44.51 \pm 16.83$ & 0.99 \\
\hline $20-39$ & 6060 & 45.44 & & 1515 & 45.44 & & \\
\hline $40-64$ & 5324 & 39.92 & & 1331 & 39.92 & & \\
\hline$>65$ & 1952 & 14.64 & & 488 & 14.64 & & \\
\hline Urbanization level & & & & & & & $<0.0001$ \\
\hline 1 (highest) & 4067 & 30.50 & & 967 & 29.00 & & \\
\hline 2 & 3845 & 28.83 & & 914 & 27.41 & & \\
\hline 3 & 2483 & 18.62 & & 576 & 17.28 & & \\
\hline 4 & 1727 & 12.95 & & 548 & 16.44 & & \\
\hline 5 (lowest) & 1214 & 9.10 & & 329 & 9.87 & & \\
\hline \multicolumn{8}{|l|}{ Comorbidities } \\
\hline Diabetes mellitus & 1288 & 9.66 & & 428 & 12.84 & & $<0.0001$ \\
\hline Hypertension & 2750 & 20.62 & & 850 & 25.49 & & $<0.0001$ \\
\hline Hyperlipidemia & 1835 & 13.76 & & 650 & 19.50 & & $<0.0001$ \\
\hline Gout & 891 & 6.68 & & 426 & 12.78 & & $<0.0001$ \\
\hline Coronary heart disease & 1444 & 10.83 & & 516 & 15.48 & & $<0.0001$ \\
\hline Chronic kidney disease & 118 & 0.88 & & 29 & 0.87 & & 0.934 \\
\hline Osteoporosis & 675 & 5.06 & & 323 & 9.69 & & $<0.0001$ \\
\hline
\end{tabular}


coronary heart disease, and osteoporosis was significantly higher among patients with AS.

Table 2 displays uni- and multivariate Cox's proportional hazard models in the AS versus nonAS cohorts between 2000 and 2011. Compared to non-AS cohort, AS cohort had a higher risk of nephrolithiasis [unadjusted hazard ratio, 1.25; 95\% CI, 1.06-1.47 ( $p=0.0067)]$. According to the result of the multivariate Cox model which controlled eight potential confounders including age, sex, urbanization level, diabetes mellitus, hypertension, hyperlipidemia, coronary heart disease, and chronic kidney disease, AS remained associated with higher risk of nephrolithiasis [adjusted hazard ratio, 1.19; 95\% CI, 1.01-1.40 $(p=0.0 .356)]$. Particularly males had a higher risk of nephrolithiasis (hazard ratio 1.92) than females.

The overall incidence rate of nephrolithiasis was higher in the AS cohort than the non-AS cohort. Additionally, males (10.10 per 1000 person-years) had a higher incidence rate of nephrolithiasis than females (6.36 per 1000 person-years) (Table 3). The Kaplan-Meier analysis was conducted to compare the accumulative incidence of nephrolithiasis between the AS and the non-AS cohorts within follow-up periods of mean 6.78 and 6.75 years. Log rank test result showed a higher incidence rate

Table 2. Cox model measured hazard ratio and 95\% confidence intervals of nephrolithiasis associated with patients with ankylosing spondylitis

\begin{tabular}{|c|c|c|c|c|c|c|c|}
\hline \multirow[t]{2}{*}{ Characteristics } & \multirow{2}{*}{$\frac{\text { Nephrolithiasis no. }(\mathrm{n}=803)}{\mathrm{n}}$} & \multicolumn{3}{|c|}{ Crude } & \multicolumn{3}{|c|}{ Adjusted } \\
\hline & & $\mathrm{HR}$ & $(95 \% \mathrm{CI})$ & $p$ & HR & $(95 \% \mathrm{CI})$ & $p$ \\
\hline \multicolumn{8}{|c|}{ Ankylosing spondylitis } \\
\hline No & 611 & 1.00 & reference & & 1.00 & reference & \\
\hline Yes & 192 & 1.25 & $(1.06-1.47)$ & 0.0067 & 1.19 & $(1.01-1.40)$ & 0.0356 \\
\hline \multicolumn{8}{|l|}{ Sex } \\
\hline Female & 249 & 1.00 & reference & & 1.00 & reference & \\
\hline Male & 554 & 0.6 & $(0.52-0.69)$ & $<0.0001$ & 1.92 & $(1.65-2.24)$ & $<.0001$ \\
\hline \multicolumn{8}{|l|}{ Age (years) } \\
\hline $20-39$ & 312 & 1.00 & reference & & 1.00 & reference & \\
\hline $40-64$ & 379 & 1.42 & $(1.22-1.65)$ & $<0.0001$ & 1.36 & $(1.15-1.6)$ & 0.0003 \\
\hline$>65$ & 112 & 1.26 & $(1.01-1.56)$ & 0.0391 & 0.99 & $(0.76-1.28)$ & 0.9081 \\
\hline \multicolumn{8}{|c|}{ Urbanization level } \\
\hline 1 (highest) & 242 & 1.00 & reference & & 1.00 & reference & \\
\hline 2 & 224 & 0.98 & (0.81-1.17) & 0.7846 & 0.96 & $(0.8-1.15)$ & 0.6799 \\
\hline 3 & 145 & 0.99 & $(0.81-1.22)$ & 0.9403 & 0.98 & $(0.8-1.21)$ & 0.8575 \\
\hline 4 & 108 & 0.99 & (0.79-1.24) & 0.936 & 0.95 & (0.76-1.19) & 0.6646 \\
\hline 5 (lowest) & 84 & 1.15 & $(0.90-1.47)$ & 0.2775 & 1.09 & $(0.85-1.4)$ & 0.5065 \\
\hline \multicolumn{8}{|c|}{ Comorbidity } \\
\hline \multicolumn{8}{|c|}{ Diabetes mellitus } \\
\hline No & 697 & 1.00 & reference & & 1.00 & reference & \\
\hline Yes & 106 & 1.43 & $(1.17-1.76)$ & 0.0006 & 1.04 & $(0.82-1.31)$ & 0.7568 \\
\hline \multicolumn{8}{|l|}{ Hypertension } \\
\hline No & 572 & 1.00 & reference & & 1.00 & reference & \\
\hline Yes & 231 & 1.60 & $(1.37-1.86)$ & $<0.0001$ & 1.43 & $(1.17-1.75)$ & 0.0005 \\
\hline \multicolumn{8}{|c|}{ Hyperlipidemia } \\
\hline No & 631 & 1.00 & reference & & 1.00 & reference & \\
\hline Yes & 172 & 1.66 & $(1.41-1.97)$ & $<0.0001$ & 1.41 & $(1.16-1.73)$ & 0.0008 \\
\hline \multicolumn{8}{|l|}{ Gout } \\
\hline No & 698 & 1.00 & reference & & & & \\
\hline Yes & 105 & 1.96 & $(1.6-2.41)$ & $<0.0001$ & & & \\
\hline \multicolumn{8}{|c|}{ Coronary heart disease } \\
\hline No & 680 & 1.00 & reference & & 1.00 & reference & \\
\hline Yes & 123 & 1.47 & $(1.21-1.78)$ & $<0.0001$ & 1.11 & $(0.88-1.39)$ & 0.3821 \\
\hline \multicolumn{8}{|c|}{ Chronic kidney disease } \\
\hline No & 798 & 1.00 & reference & & 1.00 & reference & \\
\hline Yes & 5 & 0.89 & $(0.37-2.15)$ & 0.7964 & 0.61 & $(0.25-1.48)$ & 0.2716 \\
\hline \multicolumn{8}{|l|}{ Osteoporosis } \\
\hline No & 742 & 1.00 & reference & & & & \\
\hline Yes & 61 & 1.38 & $(1.07-1.8)$ & 0.015 & & & \\
\hline
\end{tabular}


Table 3. Incidence rates, hazard ratio, and confidence intervals of nephrolithiasis for patients with and without ankylosing spondylitis stratified by demographics and comorbidities

\begin{tabular}{|c|c|c|c|c|c|c|c|c|}
\hline & \multicolumn{3}{|c|}{ Non-AS patients $(n=13,336)$} & \multicolumn{3}{|c|}{ AS patients $(n=3,334)$} & \multirow{2}{*}{$\begin{array}{c}\text { Crude HR } \\
(95 \% \text { CI })\end{array}$} & \multirow{2}{*}{$\begin{array}{c}\text { Adjusted HR } \\
(95 \% \mathrm{CI})\end{array}$} \\
\hline & Event & Person years & IR† & Event & Person years & $\mathrm{IR} \dagger$ & & \\
\hline Total & 611 & 90002 & 6.79 & 192 & 22613.65 & 8.49 & $1.25(1.06-1.47)^{* *}$ & $1.19(1.01-1.40)^{*}$ \\
\hline \multicolumn{9}{|l|}{ Sex } \\
\hline Female & 187 & 38601 & 4.84 & 62 & 9745 & 6.36 & $1.32(0.99-1.75)$ & $1.17(0.88-1.57)$ \\
\hline Male & 424 & 51400 & 8.25 & 130 & 12869 & 10.10 & $1.22(1.01-1.49)^{*}$ & $1.14(0.94-1.40)$ \\
\hline \multicolumn{9}{|l|}{ Age, (years) } \\
\hline $20-39$ & 237 & 41979 & 5.65 & 75 & 10595 & 7.08 & $1.25(0.97-1.63)$ & $1.18(0.91-1.54)$ \\
\hline $40-64$ & 285 & 36106 & 7.89 & 94 & 8967 & 10.48 & $1.33(1.05-1.68)^{*}$ & $1.21(0.95-1.53)$ \\
\hline$>65$ & 89 & 11916 & 7.47 & 23 & 3051 & 7.54 & $1.02(0.64-1.61)$ & $1.00(0.63-1.58)$ \\
\hline \multicolumn{9}{|c|}{ Urbanization level } \\
\hline 1 (highest) & 181 & 27515 & 6.58 & 61 & 6556 & 9.30 & $1.41(1.06-1.89)^{*}$ & $1.32(0.98-1.76)$ \\
\hline 2 & 179 & 26068 & 6.87 & 45 & 6272 & 7.17 & 1.05 (0.75-1.45) & 0.99 (0.71-1.38) \\
\hline 3 & 116 & 16695 & 6.95 & 29 & 3863 & 7.51 & $1.08(0.72-1.62)$ & $1.02(0.67-1.53)$ \\
\hline 4 & 74 & 11627 & 6.36 & 34 & 3728 & 9.12 & $1.44(0.96-2.16)$ & $1.28(0.84-1.93)$ \\
\hline 5 (lowest) & 61 & 8097 & 7.53 & 23 & 2195 & 10.48 & $1.39(0.86-2.25)$ & $1.35(0.83-2.20)$ \\
\hline \multicolumn{9}{|l|}{ Comorbidity } \\
\hline No & 356 & 62392 & 5.71 & 80 & 13045 & 6.13 & 1.07 (0.84-1.37) & $1.08(0.85-1.38)$ \\
\hline Yes & 255 & 27610 & 9.24 & 112 & 9569 & 11.70 & $1.27(1.02-1.59)^{*}$ & $1.24(0.99-1.55)$ \\
\hline
\end{tabular}

AS: Ankylosing spondylitis; HR: Hazard ratio; IR: Incidence rates $\dagger$ per 1,000 person-years; CI: Confidence interval; Adjusted HR: Adjusted for ankylosing spondylitis, age, sex, urbanization level and comorbidity in Cox proportional hazards regression; ${ }^{*}<0.05 ;{ }^{* *}<0.01$.

of nephrolithiasis in the AS cohort than the nonAS cohort $(p=0.0080)$ (Figure 1).

\section{DISCUSSION}

In our study, the AS cohort tended to have a higher risk of nephrolithiasis than the nonAS cohort. Though the phenomenon has not been clarified, few studies have investigated kidney stones formation among AS patients. For example, factors such as hypercalciuria, disturbed calcium and vitamin D metabolism, use of anti-inflammatory drugs, and immobilization have been suggested to cause nephrolithiasis in AS patients..$^{6,12-15}$ Furthermore, according to the ultrasonographic findings of their research, Korkmaz et al. ${ }^{6}$ reported that $25 \%$ of patients with AS had renal stone formation. Also, the result of another population-based study (sample of 8,572 AS patients) in Sweden indicated that $4.0 \%$ of the AS patients and $1.8 \%$ of the general population comparators had received a diagnosis of nephrolithiasis at the end of the study period. ${ }^{16}$ Additionally, Liu et al. ${ }^{17}$ claimed that AS patients with DM or Crohn's disease were observed to have a higher risk of nephrolithiasis.

As discussed, AS is known to be associated with various comorbidities. In their study, Bremander et al. ${ }^{18}$ suggested that AS was highly associated with comorbidities such as iridocyclitis, inflammatory bowel disease as well as cardiovascular diseases. While in another study, Szabo et al. ${ }^{19}$ also stated that AS patients were more likely (at least 25\% increased risk) to be associated with cardiovascular disease than the non-AS patients. In addition, Muñoz-Ortego et al. ${ }^{20}$ argued that AS patients tended to have a higher risk of both clinical vertebral $(0.86 \%$ versus $0.41 \%)$

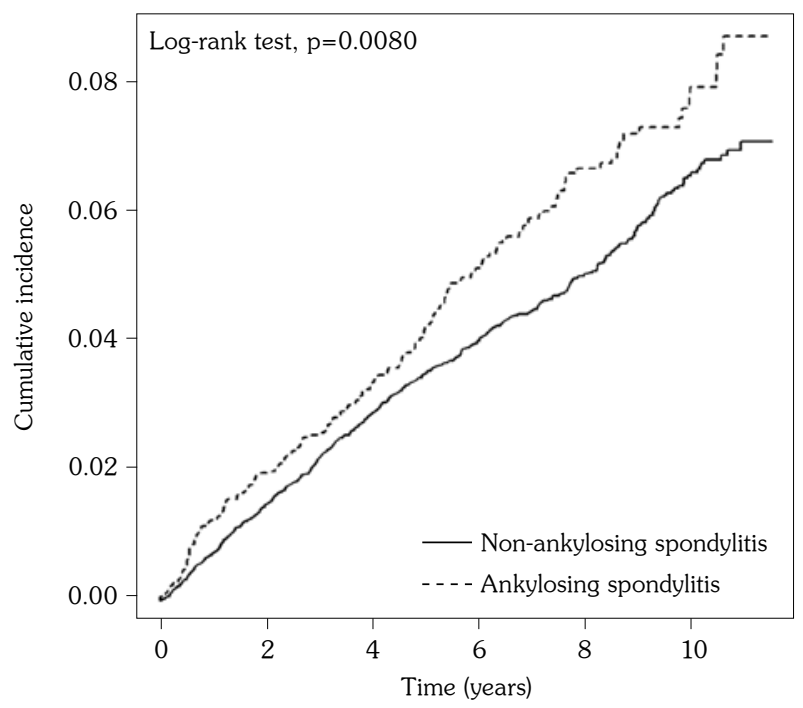

Figure 1. Estimated cumulative incidence of nephrolithiasis between ankylosing spondylitis and non-ankylosing spondylitis cohorts by Kaplan-Meier analysis. 
and nonvertebral fractures (3.4\% versus $2.7 \%$ ) than the non-AS patients. Overall, evidence of increased comorbidities such as metabolic syndrome, cardiovascular disease, gout, and osteoporosis associated with AS is consistent with the findings of our study. ${ }^{21-24}$ More interestingly, patients with above stated comorbidities appeared to have a higher risk of nephrolithiasis. ${ }^{25,26}$

Although there was a trend of increased female stone disease over the past decades, ${ }^{27}$ the male sex is still an independent risk factor of nephrolithiasis. Scales et al. ${ }^{28}$ stated that female odds ratio was 0.63 in a 16-year large scale study $(\mathrm{n}=12,110)$. While in their review, Seitz and Fajkovic ${ }^{29}$ asserted that males tended to have a higher risk of nephrolithiasis than females, with nephrolithiasis male-to-female ratio ranging from 3.1 to 1.2 in the USA and Europe. This finding is consistent with the findings of our study which male predominance in nephrolithiasis was observed in both the AS and non-AS cohorts (male-to-female ratio 2.1 and 2.27, respectively) and this further validated the fact that males with or without AS tend to have a higher risk of nephrolithiasis.

The overall frequency of nephrolithiasis in our cohort study was approximately $4.8 \%$, which was lower than the figures of other previous studies conducted in Taiwan..$^{30,31}$ One possible reason for such a low figure might be the fact that only newly diagnosed cases of nephrolithiasis from 2000 to 2011 were included in our research. Also, since only data of actual visits to medical institutions were evaluated in our research, the prevalence of nephrolithiasis in our study may have been underestimated.

As stated earlier, data used in this populationbased study were collected from the NHIRD. The use of such a database lowered the cost of our research, while at the same time, allowing us to acquire a large sample size over a relatively long time span. In addition, since this study was performed in Taiwan, of which $98 \%$ of residents are Han Chinese, the homogeneity of our sample population kept our research free from racial bias.

Despite the significant findings we obtained from our research, there are still some limitations of this study. First, many important information such as diet habits, cigarette or alcohol consumption, body weight, and family history of systemic disease was not disclosed in the NHIRD. Second, the severity and disease duration of AS could not be evaluated simply by using the ICD-9-CM coding. Third, the retrospective data generally has more confounding variables than the prospective clinical trials. Nevertheless, given the high prevalence of stone diseases in Taiwan, we hope our research offers insight into the study of nephrolithiasis in AS subgroup.

In conclusion, to our knowledge, our research is the largest Han Chinese study which assessed the risk of nephrolithiasis among AS patients compared to matched general population based on a nationwide data. Our results showed that patients with AS are more likely to be associated with nephrolithiasis than non-AS patients.

\section{Declaration of conflicting interests}

The authors declared no conflicts of interest with respect to the authorship and/or publication of this article.

\section{Funding}

This study is supported by Taiwan Ministry of Health and Welfare Clinical Trial and Research Center of Excellence (MOHW105-TDU-B-212-133019), China Medical University Hospital, Academia Sinica Taiwan Biobank Stroke Biosignature Project (BM10501010037), NRPB Stroke Clinical Trial Consortium (MOST 1042325-B-039 -005), Tseng-Lien Lin Foundation, Taichung, Taiwan, Taiwan Brain Disease Foundation, Taipei, Taiwan, and Katsuzo and Kiyo Aoshima Memorial Funds, Japan.

\section{REFERENCES}

1. Braun J, Sieper J. Ankylosing spondylitis. Lancet 2007;369:1379-90.

2. Wen YF, Wei JC, Hsu YW, Chiou HY, Wong HS, Wong $\mathrm{RH}$, et al. rs10865331 associated with susceptibility and disease severity of ankylosing spondylitis in a Taiwanese population. PLoS One 2014;9:104525.

3. Dean LE, Jones GT, MacDonald AG, Downham C, Sturrock RD, Macfarlane GJ. Global prevalence of ankylosing spondylitis. Rheumatology (Oxford) 2014;53:650-7.

4. Long LO, Park S. Update on nephrolithiasis management. Minerva Urol Nefrol 2007;59:317-25.

5. Teichman JM. Clinical practice. Acute renal colic from ureteral calculus. N Engl J Med 2004;350:684-93.

6. Korkmaz C, Ozcan A, Akçar N. Increased frequency of ultrasonographic findings suggestive of renal stones in patients with ankylosing spondylitis. Clin Exp 
Rheumatol 2005;23:389-92.

7. Pardi DS, Tremaine WJ, Sandborn WJ, McCarthy JT. Renal and urologic complications of inflammatory bowel disease. Am J Gastroenterol 1998;93:504-14.

8. Tsai MJ, Chen YT, Ou SM, Shin CJ, Peng KP, Tang $\mathrm{CH}$, et al. Increased risk of urinary calculi in patients with migraine: a nationwide cohort study. Cephalalgia 2015;35:652-61.

9. Chou PS, Kuo CN, Hung KS, Chang WC, Liao YC, Chi $\mathrm{YC}$, et al. Osteoporosis and the risk of symptomatic nephrolithiasis: a population-based 5-year follow-up study in Taiwan. Calcif Tissue Int 2014;95:317-22.

10. Chen HS, Su LT, Lin SZ, Sung FC, Ko MC, Li CY. Increased risk of urinary tract calculi among patients with diabetes mellitus--a population-based cohort study. Urology 2012;79:86-92.

11. Lin YJ, Tian WH, Chen CC. Urbanization and the utilization of outpatient services under National Health Insurance in Taiwan. Health Policy 2011;103:236-43.

12. Arends S, Spoorenberg A, Bruyn GA, Houtman PM, Leijsma MK, Kallenberg CG, et al. The relation between bone mineral density, bone turnover markers, and vitamin $\mathrm{D}$ status in ankylosing spondylitis patients with active disease: a cross-sectional analysis. Osteoporos Int 2011;22:1431-9.

13. Canalis E, Mazziotti G, Giustina A, Bilezikian JP. Glucocorticoid-induced osteoporosis: pathophysiology and therapy. Osteoporos Int 2007;18:1319-28.

14. Stewart AF, Adler M, Byers CM, Segre GV, Broadus AE. Calcium homeostasis in immobilization: an example of resorptive hypercalciuria. $\mathrm{N}$ Engl J Med 1982;306:1136-40.

15. Worcester EM, Coe FL. Clinical practice. Calcium kidney stones. N Engl J Med 2010;363:954-63.

16. Jakobsen AK, Jacobsson LT, Patschan O, Askling J, Kristensen LE. Is nephrolithiasis an unrecognized extra-articular manifestation in ankylosing spondylitis? A prospective populationbased Swedish national cohort study with matched general population comparator subjects. PLoS One 2014;9:113602.

17. Lui NL, Carty A, Haroon N, Shen H, Cook RJ, Inman RD. Clinical correlates of urolithiasis in ankylosing spondylitis. J Rheumatol 2011;38:1953-6.

18. Bremander A, Petersson IF, Bergman S, Englund M. Population-based estimates of common comorbidities and cardiovascular disease in ankylosing spondylitis.
Arthritis Care Res (Hoboken) 2011;63:550-6.

19. Szabo SM, Levy AR, Rao SR, Kirbach SE, Lacaille D, Cifaldi $\mathrm{M}$, et al. Increased risk of cardiovascular and cerebrovascular diseases in individuals with ankylosing spondylitis: a population-based study. Arthritis Rheum 2011;63:3294-304.

20. Muñoz-Ortego J, Vestergaard P, Rubio JB, Wordsworth $\mathrm{P}$, Judge A, Javaid MK, et al. Ankylosing spondylitis is associated with an increased risk of vertebral and nonvertebral clinical fractures: a population-based cohort study. J Bone Miner Res 2014;29:1770-6.

21. Ho HH, Chen JY. Ankylosing spondylitis: Chinese perspective, clinical phenotypes, and associated extra-articular systemic features. Curr Rheumatol Rep 2013;15:344.

22. Ho HH, Yu KH, Chen JY, Lin JL, Wu YJ, Luo SF, et al. Coexisting ankylosing spondylitis and gouty arthritis. Clin Rheumatol 2007;26:1655-61.

23. Kang JH, Chen YH, Lin HC. Comorbidity profiles among patients with ankylosing spondylitis: a nationwide population-based study. Ann Rheum Dis 2010;69:1165-8.

24. Incel NA, Gökoğlu F, Nacir B, Incel N. Bone and stone in ankylosing spondylitis: osteoporosis and urolithiasis. Clin Rheumatol 2006;25:667-70.

25. Rendina D, De Filippo G, D'Elia L, Strazzullo P. Metabolic syndrome and nephrolithiasis: a systematic review and meta-analysis of the scientific evidence. $\mathrm{J}$ Nephrol 2014;27:371-6.

26. Lange JN, Mufarrij PW, Wood KD, Holmes RP, Assimos DG. The association of cardiovascular disease and metabolic syndrome with nephrolithiasis. Curr Opin Urol 2012;22:154-9.

27. Marickar YM, Vijay A. Female stone disease: the changing trend. Urol Res 2009;37:337-40.

28. Scales CD Jr, Smith AC, Hanley JM, Saigal CS. Prevalence of kidney stones in the United States. Eur Urol 2012;62:160-5.

29. Seitz C, Fajkovic H. Epidemiological gender-specific aspects in urolithiasis. World J Urol 2013;31:1087-92.

30. Huang WY, Chen YF, Carter S, Chang HC, Lan CF, Huang $\mathrm{KH}$. Epidemiology of upper urinary tract stone disease in a Taiwanese population: a nationwide, population based study. J Urol 2013;189:2158-63.

31. Chen YK, Lin HC, Chen CS, Yeh SD. Seasonal variations in urinary calculi attacks and their association with climate: a population based study. J Urol 2008;179:564-9. 